\title{
Design and Development of a Test Rig for 13.56 MHz IPT Systems with Synchronous Rectification and Bidirectional Capability
}

\author{
Nunzio Pucci, Juan M. Arteaga and Paul D. Mitcheson \\ Department of Electrical and Electronic Engineering, Imperial College London \\ np1915@ic.ac.uk
}

\begin{abstract}
This paper presents the development of a test rig for bidirectional $13.56 \mathrm{MHz}$ wireless power using identical backto-back Class EF converters. Theoretical principles of bidirectional wireless power are described and an operating chart representing the range of admissible complex voltages induced on the active transmit side is introduced. The implementation is achieved by driving the gate signals of two Class EF coildrivers from a signal generator, allowing the relative phase of the currents in each coil to be controlled. The rig sets a constant input voltage for each of the two coil-drivers by implementing a source-sink configuration, emulating a bidirectional DC-DC conversion stage at each side. This setup can also be used to test for tuning mismatches and different loading conditions in the back-to-back Class EF configuration. Experimental results include bidirectional wireless power transmission of $20 \mathrm{~W}$ across a $13.56 \mathrm{MHz}$ link with $6.56 \%$ coupling. The combination of low coupling factors and moderate power levels enables new classes of applications that require large air gaps and tolerance to misalignment such as in micro e-mobility. High efficiency can be maintained despite changes in coupling factors and load since active rectification ensures operation at the resonant point in both tanks.
\end{abstract}

\section{INTRODUCTION}

Bidirectional wireless power transfer is an increasingly growing topic of research, with applications ranging from low-power battery charging for drones [1] to high-power EVto-grid applications [2]. In inductive power transfer (IPT) for unmanned vehicles, bidirectional operation is a convenient feature which allows a system to either provide power to other devices or recharge its battery without the need of human interaction.

One of the key aspects to achieve bidirectionality in IPT systems is synchronisation: for a system to operate either as an inverter or a rectifier, the coil current needs to be respectively at a phase of $0^{\circ}$ or $180^{\circ}$ with respect to the induced voltage produced by the other coil, and thus operate at unity power factor (i.e. no reflected reactances) [3]. Active rectification allows operation of both tanks at resonance even with varying coupling factors and loads, enabling operation at coupling factors as low as $5 \%$. Research has been done for low-frequency bidirectional systems, e.g. [2], [4]. The recent efforts in synchronous high frequency inductive power transfer (HF-IPT) systems have also led to the formulation of interesting solutions based on the processing of the receiver's waveforms, e.g. [5], [6]. Applications for high coupling

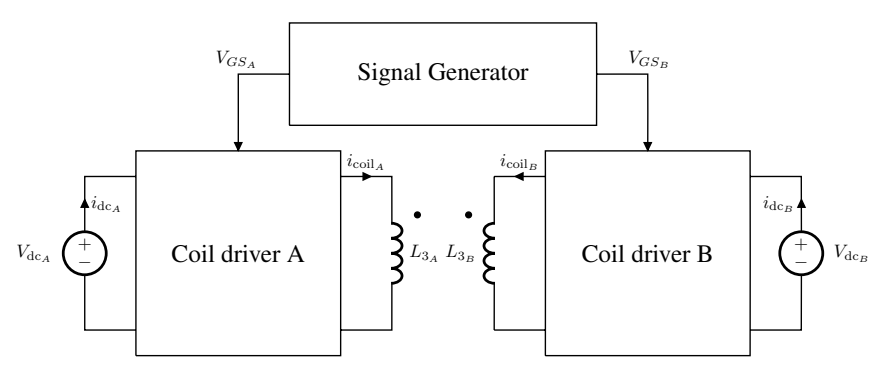

Fig. 1. Block diagram of the test rig

(above 10\%) bidirectional HF-IPT systems can be found in [7], [8].

One of the differences of HF-IPT with respect to systems working in the kilohertz range is the possibility of designing a system that does not necessarily use magnetically permeable materials. This is due to the improvement in quality factor of low inductance coils $(<5 \mu \mathrm{H})$ in the megahertz range [9][12], which is around 760 in the presented work [13]. A direct consequence of operating in this frequency range is the possibility of integration of compact light-weight systems in a wide number of applications, ranging from consumer electronics to healthcare and military.

In this paper a solution is shown to assess a bidirectional HF-IPT system with emulation of an ideal synchronisation technique and bidirectional DC-DC block obtained through a source-sink configuration of electronic load and DC power supply. The DC-DC bidirectional blocks could also be practically achieved as described in [14]. The presented test rig allows the assessment and optimisation of the system to be focused on coil drivers and IPT links.

\section{Test Rig Configuration}

The test rig is comprised of two identical transceivers as shown in Fig. 1 and Fig. 2.

The two sides are driven by a common dual channel signal generator and two independent voltage sources (equipment models specified in Table I). The voltage source $V_{\mathrm{dc}}$ of the secondary is connected in parallel with an electronic load operating in constant voltage (CV) mode to obtain a sinksource configuration. This step allows each side to be operated 
TABLE I

SUMMARY OF EQUIPMENT

\begin{tabular}{cc}
\hline \hline Instrument & Manufacturer, model and specs \\
\hline Signal generator & Aim TTI, TG5011A, dual channel 50 MHz \\
Oscilloscopes & Teledyne Lecroy, HDO4034A, 4 Channels 2.5 GS/s and Teledyne Lecroy, HDO6104A, 4 Channels 10 GS/s \\
Probes & Voltage: Passive Lecroy probes - PMK, PHV 1000 100:1; Curent: Keysight, N2783B \\
$\begin{array}{c}\text { Digital Power Meter } \\
\text { DC Power Supplies }\end{array}$ & Agilent Technologies, N8741A (0-300 V, 0-11 A) - Agilent Technologies,U8032A (0-60 V, 0-3 A) for current source and gate drivers \\
Electronic Load & BK Precision, BK8512 (0-500 V, 0-240 A $)$
\end{tabular}

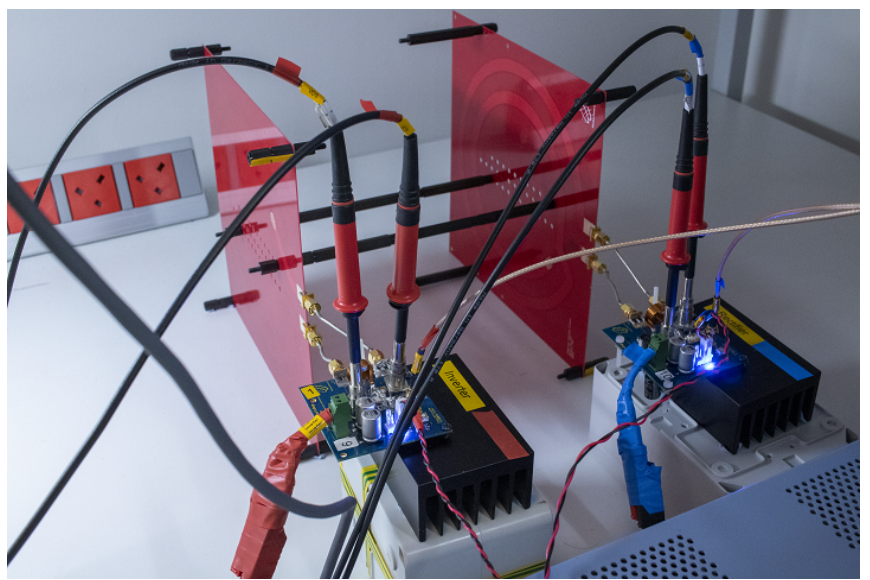

(a) IPT Link

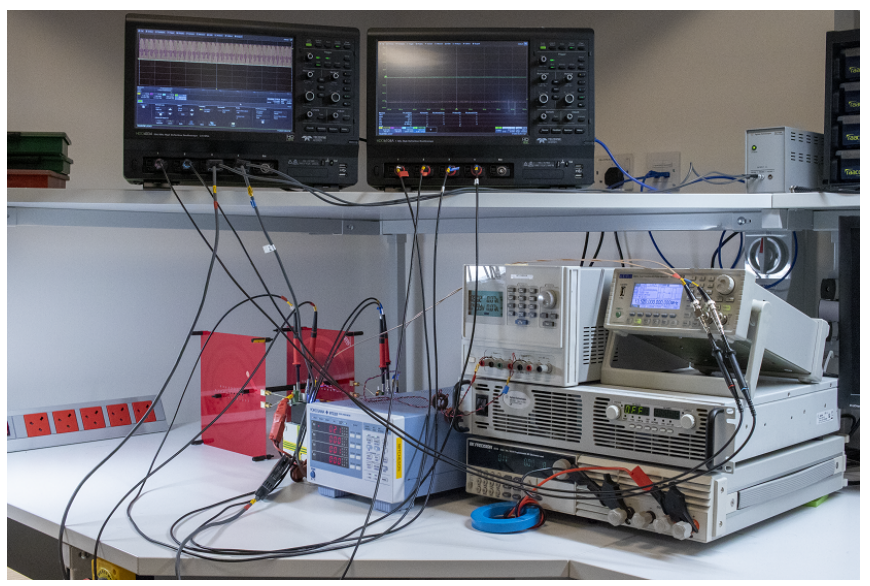

(b) Setup including instrumentation

Fig. 2. Test rig pictures

under a regulated DC input voltage, granting the transceivers the option of working in bidirectional mode.

\section{Design of a Bidirectional CoIl Driver Using CLASS EF CIRCUITS}

The Class EF topology (both transceivers in Fig. 3) is a commonly employed HF-IPT coil driver due to its high efficiency at $\mathrm{MHz}$ frequencies [15], [16] and its convenience of only comprising one low side transistor.
This topology is usually designed to operate at constant duty cycle and frequency (i.e. in open loop), requiring power throughput control to be implemented in the previous or the latter stages of the system. This topology can be employed as an inverter or a rectifier [17], [18], where the direction of the power flow is determined by the relative polarity of the magnetic link and the relative phase $\left( \pm 90^{\circ}\right)$ of the coil currents.

In [18] a load-independent design formulation for this topology was shown where the transistor drain voltage converges to zero at turn-on independent of the resistive coupled load. In this work we expand that principle to allow for backto-back circuits using this topology, and show that this loadindependent tuning can not only operate as load-independent inverters but as bidirectional coil drivers that can change the direction of the power flow by changing the relative phase of the driving signals of the transceivers by $180^{\circ}$ (from $90^{\circ}$ to $-90^{\circ}$ or conversely).

\section{Optimisation of The Relative Phase}

In a system with two coupled ideal inductors (Fig. 4 (a)) with self inductances of $L_{3_{\mathrm{A}}}$ and $L_{3_{\mathrm{B}}}$, the voltage across the primary $L_{3_{\mathrm{A}}}$ ) can be obtained as:

$$
v_{\mathrm{coil}_{\mathrm{A}}}=L_{3_{\mathrm{A}}} \frac{\mathrm{d} i_{\text {coil }_{\mathrm{A}}}}{\mathrm{d} t}+M_{\mathrm{AB}} \frac{\mathrm{d} i_{\mathrm{coil}_{\mathrm{B}}}}{\mathrm{d} t},
$$

with $i_{\text {coil }_{\mathrm{A}}}$ and $i_{\text {coil }_{\mathrm{B}}}$ being the currents in coils $L_{3_{\mathrm{A}}}$ and $L_{3_{\mathrm{B}}}$ respectively, and $M_{\mathrm{AB}}$ being the mutual inductance between them. It is possible to rewrite the system at the primary side by including the induced voltage by the secondary $\left(v_{\mathrm{M}}\right)$ as a function of the secondary coil current. This is shown in (Fig. 4 (b)). The equations for this rearranged system representation are given by:

$$
v_{\mathrm{coil}_{\mathrm{A}}}=L_{3_{\mathrm{A}}} \frac{\mathrm{d} i_{\mathrm{coil}_{\mathrm{A}}}}{\mathrm{d} t}+v_{\mathrm{M}},
$$

and $v_{\mathrm{M}}$ in (1) can be rewritten to include the coupling factor $\left(k_{\mathrm{AB}}\right)$ :

$$
v_{\mathrm{M}}=M_{\mathrm{AB}} \frac{\mathrm{d} i_{\mathrm{coil}_{\mathrm{B}}}}{\mathrm{d} t}=\left(k_{\mathrm{AB}} \sqrt{L_{3_{\mathrm{A}}} L_{3_{\mathrm{B}}}}\right) \frac{\mathrm{d} i_{\mathrm{coil}_{\mathrm{B}}}}{\mathrm{d} t} .
$$

In phasor notation ${ }^{1}$ Equation (3) can be formulated as:

$$
\boldsymbol{v}_{\mathrm{M}}=\boldsymbol{j} 2 \pi f_{\mathrm{i}_{\mathrm{coil}_{\mathrm{B}}}} k_{\mathrm{AB}} \sqrt{L_{3_{\mathrm{A}} L_{3_{\mathrm{B}}}}} \boldsymbol{c}_{\text {coil }_{\mathrm{B}}},
$$

\footnotetext{
${ }^{1}$ Phasors are presented in bold.
} 


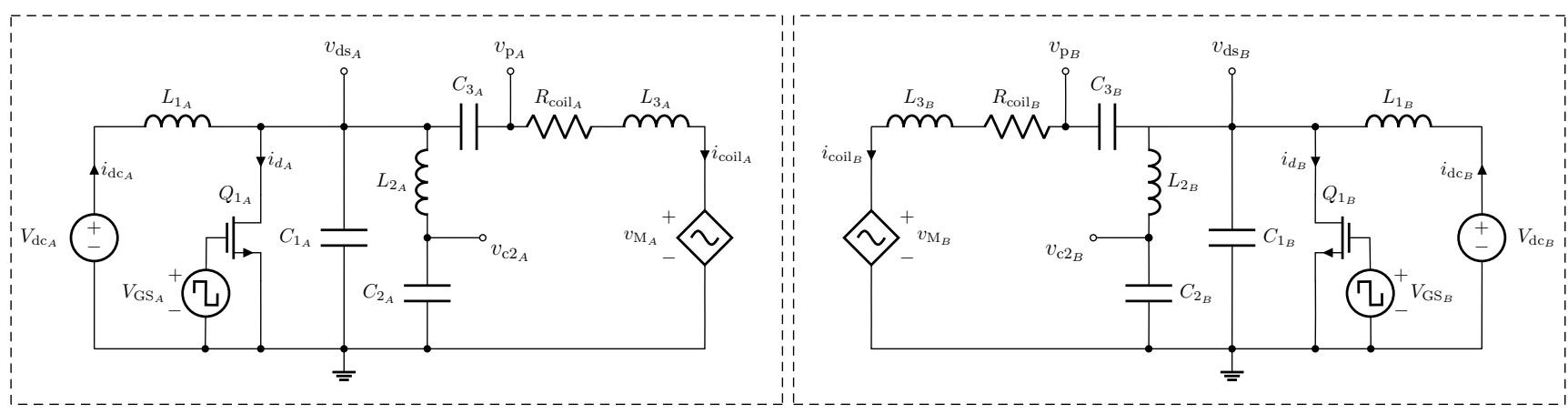

Fig. 3. Circuit diagram of two bidirectional Class EF inverters with the loads modelled as dependent voltage sources.
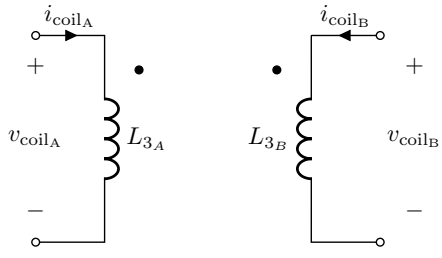

(a) Two-port representation of the link

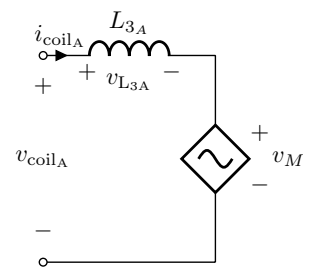

(b) Primary coil equivalent circuit including induced voltage

Fig. 4. Circuit diagrams of inductively coupled ideal coils.

assuming sinusoidal currents in the coils with frequencies $f_{\mathrm{i}_{\mathrm{coil}_{\mathrm{A}}}}=f_{\mathrm{i}_{\mathrm{coil}_{\mathrm{B}}}}$.

Rearranging (4) in function of the magnitudes of coil currents $i_{\text {coil }_{\mathrm{A}}}$ and $i_{\text {coil }_{\mathrm{B}}}$ and the phase angle of $\boldsymbol{i}_{\text {coil }_{\mathrm{B}}}\left(\theta_{\mathrm{B}}\right)$ we obtain:

$$
\begin{aligned}
\boldsymbol{v}_{\mathrm{M}}= & -2 \pi f_{\mathrm{i}_{\text {coil }_{\mathrm{A}}}} k_{\mathrm{AB}} \sqrt{L_{3_{\mathrm{A}}} L_{3_{\mathrm{B}}}} i_{\mathrm{coil}_{\mathrm{B}}} \sin \left(\theta_{\mathrm{B}}\right) \\
& +\boldsymbol{j}\left[2 \pi f_{\mathrm{i}_{\mathrm{coil}_{\mathrm{A}}}} k_{\mathrm{AB}} \sqrt{L_{3_{\mathrm{A}}} L_{3_{\mathrm{B}}}} i_{\mathrm{coil}_{\mathrm{B}}} \cos \left(\theta_{\mathrm{B}}\right)\right] \\
= & 2 \pi f_{\mathrm{i}_{\mathrm{coil}_{\mathrm{A}}}} k_{\mathrm{AB}} \sqrt{L_{3_{\mathrm{A}}} L_{3_{\mathrm{B}}}} i_{\text {coil }_{\mathrm{B}}} / \theta_{\mathrm{B}}+90^{\circ} .
\end{aligned}
$$

From (4) it can be inferred that the real part of $\boldsymbol{v}_{\mathbf{M}}$ is maximised for $\theta_{\mathrm{B}}=-90^{\circ}$ and minimised for $\theta_{\mathrm{B}}=90^{\circ}$. These two cases correspond to side $\mathrm{B}$ operating respectively as a receiver and a transmitter with unity power factor.

It is worth noting that this corresponds to the case in which both sides are at resonance. When this condition is not satisfied (assuming side A is tuned and side B is detuned), the required value of $\theta_{\mathrm{B}}$ such that side $\mathrm{B}$ works as a rectifier with unity power factor will be $\theta_{\mathrm{B}}<-90^{\circ}$ when the resonant tank of side $\mathrm{B}$ is too capacitive and $\theta_{\mathrm{B}}>-90^{\circ}$ when the resonant tank of side $\mathrm{B}$ is too inductive. A similar process applies for side B working as a transmitter.

\section{ANAlysis of Operating Chart BASED on Induced VOLTAGE}

Before operating the system it is necessary to determine the feasible range of operating conditions of the transceivers. This type of assessment can be performed with a wide number

of criteria, ranging from voltage and current capability of the components to overall system stability.

In the experimental section of this work the coil drivers were constructed using $650 \mathrm{~V}, 15 \mathrm{~A} \mathrm{GaN}$ transistors (GS66504B), the component values of the inverter used in [1], and a fixed $V_{\mathrm{dc}}$ of $60 \mathrm{~V}$ (the boards are shown in Fig. 2). With respect to the admissible load range, expressed in terms of the magnitude and phase of the induced voltage, a thermal boundary was set as the device temperature reaches $70^{\circ} \mathrm{C}$. The operating chart based on induced voltage is illustrated in Fig. 5. The two main cases that contribute to power losses are when the drain-voltage is different than zero at turn-on (hardswitching in Fig. 5) and reverse conduction of the device. There are also cases of low-frequency oscillation in proximity of the hard-switching conditions where the temperature did not reach the $70^{\circ} \mathrm{C}$ limit, however these results were not included in the admissible induced voltage range.

It is possible to notice an unbalance in positive and negative portions for the feasible scenarios boundary, which suggest a slight detuning of the resonant tank, which is expected since the converters are operating at a lower voltage than their original design $(77 \mathrm{~V})$, thus slightly changing the tuning due to the transistors nonlinear $C_{\mathrm{O}_{\mathrm{ss}}}$.

\section{EXPERIMENTAL VALIDATION}

The same transceivers (A and B) are used in six different scenarios to show the system operating as an inverter and as an active rectifier for a fixed coupling of $6.56 \%$ and varying power factors. The system manages to transfer an average of $21.5 \mathrm{~W}$ with and efficiency of $71.1 \%$ when $\theta= \pm 90^{\circ}$ (Fig. 6). In these two cases the switching waveforms of the drain node for both A and B showcase how the time-reversed signal is associated with the two separate cases of power delivery and reception. As reported in the literature [17], there is potential to achieve better efficiency with this technology for higher input voltages and power levels or by employing coils with higher $\mathrm{Q}$, however for this specific design the standby power of each transceiver is close to $5 \mathrm{~W}$, making it difficult to achieve better efficiencies in a $20 \mathrm{~W}$ system.

It is possible to observe how the $v_{\mathrm{ds}_{\mathrm{B}}}$ waveform changes in the first three plots of Fig. 6 when side B transmits power, 


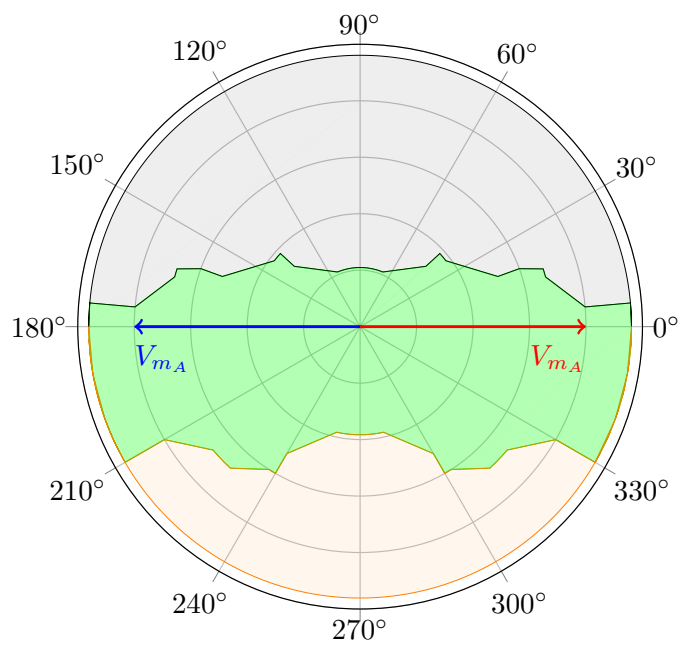

$\begin{array}{llllll}0 & 5 & 10 & 15 & 20 & 25\end{array}$

$\begin{array}{|cc|}\longrightarrow & \text { Feasible scenarios } \\ & \text { Hard-switching } \\ & \text { Reverse conduction } \\ \longrightarrow & \text { Transceiver A transmitting power } \\ \longrightarrow & \text { Transceiver A receiving power }\end{array}$

Fig. 5. Operating chart based on induced voltage magnitude and angle for a thermal boundary of $70^{\circ} \mathrm{C}$ (Transceiver A)

exhibiting hard switching for $\theta<90^{\circ}$ and approaching diode conduction for $\theta>90^{\circ}$. Hard switching is still visible in the $\theta=90^{\circ}$ case, implying that there is too much capacitance on side $\mathrm{A}$. This could be compensated with minor changes in relative phase. Similar conclusions can be drawn for the last 3 plots, however hard-switching for $\theta<-90^{\circ}$ is less noticeable.

As shown in Fig. 6 the system has a performance which is independent of the direction of power for unity power factors, since the output power and DC-DC system efficiency are practically the same for $\theta=90^{\circ}$ and $\theta=-90^{\circ}$.

\section{CONCLUSION}

In this work a test rig structure for the attainment of experimental results to evaluate the transceivers and link of a bidirectional HF-IPT system was described. The sink-source at both ends, which emulates a bidirectional DC-DC converter, and the dual channel signal generator, which provides the synchronisation between the two transceivers, allow the experiments to be focused exclusively on the performance of the transceivers and the link. Bidirectional operation of a Class EF coil driver is demonstrated at a fixed coupling of $6.56 \%$ for variable power factors and power levels up to $20 \mathrm{~W}$.

\section{ACKNOWLEDGEMENT}

The authors would like to acknowledge the following funding sources: EPSRC Converter Architectures, grant ref: EP/R004137/1; EPSRC Quietening ultra-low-loss SiC \& GaN

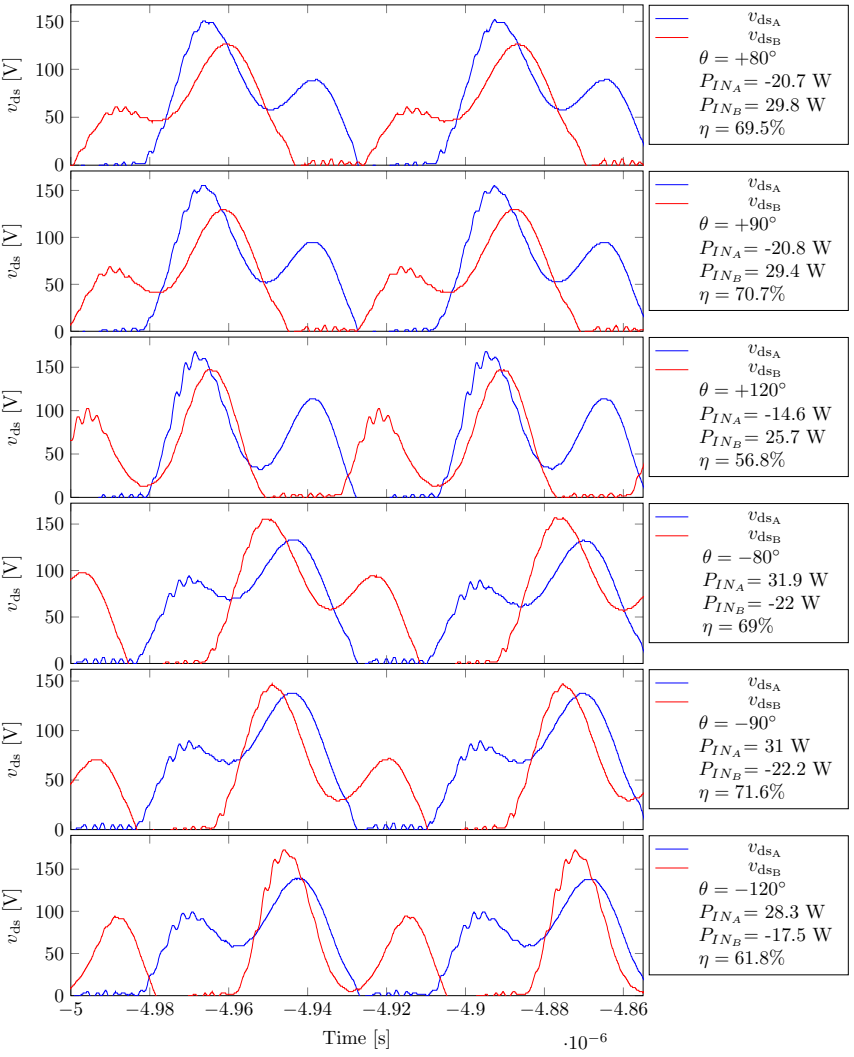

Fig. 6. Experimental $v_{\mathrm{ds}}$ waveform under different operating conditions $\left(\mathrm{V}_{\mathrm{dc}_{\mathrm{A}}}=60 \mathrm{~V}, \mathrm{~V}_{\mathrm{dc}_{\mathrm{B}}}=60 \mathrm{~V}, \mathrm{k}=6.56 \%\right)$

waveforms, grant ref: EP/R029504/1; EPSRC Impact Acceleration Account - Imperial College London, grant ref: EP/R511547/1; SitS NSF-UKRI: Wireless In-Situ Soil Sensing Network for Future Sustainable Agriculture', grant ref: NE/T011467/1; the Department of Electrical and Electronic Engineering, Imperial College London and the University of Costa Rica.

\section{REFERENCES}

[1] J. M. Arteaga, S. Aldhaher, G. Kkelis, C. Kwan, D. C. Yates, and P. D. Mitcheson, "Dynamic capabilities of multi-MHz inductive power transfer systems demonstrated with batteryless drones," IEEE Trans. on Power Electron., vol. 34, no. 6, pp. 5093-5104, June 2019.

[2] U. K. Madawala and D. J. Thrimawithana, "A bidirectional inductive power interface for electric vehicles in V2G systems," IEEE Transactions on Industrial Electronics, vol. 58, no. 10, pp. 4789-4796, 2011.

[3] J. M. Arteaga, N. Pucci, L. Lan, and P. D. Mitcheson, "Load characterisation in high frequency ipt systems using class ef switching waveforms," IEEE Trans. on Power Electron., submitted for publication, pp. 1-9, 2021

[4] D. J. Thrimawithana, U. K. Madawala, and M. Neath, "A synchronization technique for bidirectional IPT systems," IEEE Transactions on Industrial Electronics, vol. 60, no. 1, pp. 301-309, 2013.

[5] S. Cochran and D. Costinett, "Frequency Synchronization and Control for a $6.78 \mathrm{MHz}$ WPT Active Rectifier," 2018 IEEE 19th Workshop on Control and Modeling for Power Electronics, COMPEL 2018, pp. 1-7, 2018.

[6] S. Aldhaher, D. C. Yates, and P. D. Mitcheson, "Class E Rectifier using GaN devices for Space-Constrained Applications," 2018 IEEE Wireless Power Transfer Conference (WPTC), pp. 1-4, 2018. 
[7] X. Zan and A.-T. Avestruz, "27 . $12 \mathrm{MHz}$ Bi-Directional Wireless Power Transfer Using Current-Mode Class D Converters with PhaseShift Power Modulation," IEEE PELS Workshop on Emerging Technologies: Wireless Power Transfer (Wow), pp. 0-5, 2018.

[8] M. Huang, Y. Lu, S.-P. U, and R. Martins, "Transceiver with MaximumCurrent Charging Mode," Digest of Technical Papers - IEEE International Solid-State Circuits Conference, vol. 3, pp. 376-378, 2017.

[9] K. Van Schuylenbergh and R. Puers, Inductive powering: Basic theory and application to biomedical systems. Springer Science \& Business Media, 2009.

[10] A. Kurs, A. Karalis, R. Moffatt, J. D. Joannopoulos, P. Fisher, and M. Soljačić, "Wireless power transfer via strongly coupled magnetic resonances," science, vol. 317, no. 5834, pp. 83-86, 2007.

[11] S. Y. R. Hui, W. Zhong, and C. K. Lee, "A critical review of recent progress in mid-range wireless power transfer," Power Electron., IEEE Trans. on, vol. 29, no. 9, pp. 4500-4511, Sept 2014.

[12] M. Pinuela, D. C. Yates, S. Lucyszyn, and P. D. Mitcheson, "Maximizing DC-to-load efficiency for inductive power transfer," IEEE Trans. Power Electron., vol. 28, no. 5, pp. 2437-2447, May 2013.

[13] E. Skountzos, J. M. Arteaga, E. Hadjittofis, D. C. Yates, K. L. Sedransk
Campbell, and P. D. Mitcheson, "A 13.56 MHz inductive power transfer system operating with corroded coils," in IEEE PELS Workshop on Emerging Technologies: Wireless Power Transfer (WoW), 2019, pp. 335-340.

[14] M. Fu, C. Ma, and X. Zhu, "A cascaded boost-buck converter for high-efficiency wireless power transfer systems," IEEE Transactions on Industrial Informatics, vol. 10, no. 3, pp. 1972-1980, 2014.

[15] J. Choi, D. Tsukiyama, Y. Tsuruda, and J. Rivas, "13.56 MHz $1.3 \mathrm{~kW}$ resonant converter with GaN fet for wireless power transfer," in 2015 IEEE Wireless Power Transfer Conf. (WPTC), May 2015, pp. 1-4.

[16] J. M. Arteaga, S. Aldhaher, G. Kkelis, D. C. Yates, and P. D. Mitcheson, "Multi-MHz IPT systems for variable coupling," IEEE Trans. Power Electron., vol. 33, no. 9, pp. 7744-7758, Sept 2018.

[17] Z. Kaczmarczyk, "High-efficiency Class E, $E F_{2}$, and $E / F_{3}$ inverters," IEEE Trans. on Ind. Electron., vol. 53, no. 5, pp. 1584-1593, Oct 2006.

[18] S. Aldhaher, P. D. Mitcheson, and D. C. Yates, "Load-independent Class $\mathrm{EF}$ inverters for inductive wireless power transfer," in IEEE Wireless Power Transfer Conf. (WPTC), May 2016, pp. 1-4. 